\title{
Review: specially designed products to prevent or heal pressure sores are more effective than standard mattresses
}

Cullum N, Deeks J, Sheldon TA, et al. Beds, mattresses and cushions for pressure sore prevention and treatment. (Cochrane Review, latest version 26 May 1999). In: Cochrane Library. Oxford: Update Software.

QUESTION: In patients who are at risk of pressure sores, are pressure relieving beds, mattresses, and cushions (support surfaces) effective for preventing and treating pressure sores?

\section{Data sources}

Randomised controlled trials (RCTs) were identified by searching 19 databases from their inception to 1997 , hand searches of 5 wound care journals, conference abstracts, bibliographies of relevant studies and review articles, and contact with experts and manufacturers. Unpublished data were also sought.

\section{Study selection}

Interventions included standard or specialised foam mattresses or overlays; gel, fibre, or water filled mattresses or overlays; alternating pressure mattresses or overlays; air fluidised, bead, or low air loss beds; sheepskins; turning beds or frames; wheelchair cushions; or operating table overlays; and outcomes were incidence or healing rates of sores, cost, comfort, reliability, and acceptability.

\section{Data extraction}

Data were extracted on study quality, inclusion and exclusion criteria, baseline characteristics, settings, interventions, follow up, outcomes, acceptability, and reliability.

\section{Main results}

37 RCTs met the inclusion criteria. Methodological quality was generally poor, many settings and products were studied, and sample size was often small. 29 RCTs studied patients without pre-existing pressure sores. Special mattresses had lower rates of incidence or severity of pressure ulcers: the Comfortex DeCube mattress, Beaufort Bead bed, Softform mattress, and a water filled mattress were better than standard hospital mattresses for high risk patients (relative risks [RR] ranged from 0.2-0.35); Clinifloat, Therarest, Transfoam, and Vaperm mattresses were better than standard foam mattresses for incidence of pressure sores for all patients and those with hip fractures (pooled RR $0.29,95 \%$ CI 0.19 to 0.43 ). Low technology products (eg, foams and water filled supports) did not differ from each other for the incidence of pressure ulcers. Only 1 RCT of 7 showed that alternating pressure devices were superior to low technology constant pressure products. The 1 RCT of low air loss beds showed that they were more cost effective than a standard bed in the intensive care unit. The 2 RCTs of kinetic turning tables did not show differences. 3 RCTs evaluated special products for the operating suite; 1 evaluated a polymer pad and showed a $47 \%$ reduction in pressure sores and 2 evaluated an alternating system and showed a pooled RR of 0.21 (CI 0.06 to 0.7). The role of seat cushions in pressure sore prevention is unclear. Air fluidised beds were superior to standard beds for healing of pressure sores.

\section{Conclusions}

Special products designed to prevent or cure pressure sores are generally more effective than standard mattresses. Studies that evaluate specific products or types of products are limited in size and quality.

\section{COMMENTARY}

The body of literature about support surfaces is generally confusing. This summary of research evidence by Cullum $e t$ $a l$ is clinically important because it focuses on patient outcomes (incidence and healing rates of pressure sores). Other studies have reported interface pressure, blood flow, or transcutaneous oxygen concentrations, but failed to provide data about patient outcomes. This review updates the reviews by the US Agency for Health Care Policy and Research $^{1}$ and accurately concludes that standard hospital mattresses are consistently outperformed by several foam based, low pressure mattresses and overlays for the prevention of pressure ulcers. Initial evidence supports the superiority of higher technology devices for treating ulcers, but more research is needed.

One strength of the review is the comprehensiveness of the literature search, which included articles from the UK and US written over a period of $\geqslant 30$ years. The good news from this synthesis is that low technology support surfaces can reduce the incidence of pressure ulcers better than standard hospital mattresses, pressure reduction in the operating suite is possible, and some evidence supports the effectiveness of air fluidised and low air loss devices. The bad news is that data do not provide guidance on which specific products are more cost effective and result in fewer pressure ulcers.

One limitation in the interpretation of these data is the inadequate description of the standard hospital mattress, as well as the support surfaces to which they are compared. It is important that future reports summarising evaluations of these products include this information. Clinicians should seek evidence of pressure sore incidence or healing. Another limitation is that patient level of risk, an important covariate in any study of incidence, is not adequately addressed. Low risk patients may not need the more costly devices.

The results of this review are relevant to nurses who care for patients at risk of developing pressure ulcers in acute care hospitals, intensive care units, and nursing homes, as well as elderly people and individuals in the operating suite. Clinicians should consider which patients need pressure relief based on objective criteria. Risk assessment can inform decisions, especially when levels of mobility in bed are assessed. Advanced practice nurses, members of skin care teams, and others who deal with patients at risk need to be informed consumers and demand data to support product claims. Nancy Bergstrom, RN, PhD College of Nursing, University of Nebraska Medical Center Omaha, Nebraska, USA

1 Bergstrom N, Bennett MA, Carlson CE et al. Treatment of pressure ulcers. Clinical practice guideline No. 15; Rockville, MD: Agency for Health Care Policy and Research; 1994.
A modified version of this abstract appears in ACP Journal Club. 